\title{
Bu open Two-group randomised, parallel trial of cognitive and exposure therapies for problem gambling: a research protocol
}

\author{
David P Smith, ${ }^{1}$ Malcolm W Battersby, ${ }^{1}$ Peter W Harvey, ${ }^{1}$ Rene G Pols, ${ }^{1}$ \\ Robert Ladouceur ${ }^{2}$
}

To cite: Smith DP,

Battersby MW, Harvey PW, et al. Two-group randomised, parallel trial of cognitive and exposure therapies for problem gambling: a research protocol. BMJ Open 2013;3: e003244. doi:10.1136/ bmjopen-2013-003244

- Prepublication history for this paper is available online. To view these files please visit the journal online (http://dx.doi.org/10.1136/ bmjopen-2013-003244).

Received 17 May 2013 Revised 22 May 2013 Accepted 29 May 2013

This final article is available for use under the terms of the Creative Commons Attribution Non-Commercial 3.0 Licence; see http://bmjopen.bmj.com

\footnotetext{
${ }^{1}$ Department of Psychiatry, Flinders Human Behaviour and Health Research Unit, Flinders University, Adelaide, South Australia, Australia ${ }^{2}$ School of Psychology, Université Laval, Québec, Canada
}

\section{Correspondence to} David P Smith; david.smith@flinders.edu.au

\begin{abstract}
Background: Problem gambling is a serious public health concern at an international level where population prevalence rates average $2 \%$ or more and occurs more frequently in younger populations. The most empirically established treatments until now are combinations of cognitive and behavioural techniques labelled cognitive behaviour therapy (CBT). However, there is a paucity of high quality evidence for the comparative efficacy of core CBT interventions in treating problem gamblers. This study aims to isolate and compare cognitive and behavioural (exposurebased) techniques to determine their relative efficacy.

Methods: A sample of 130 treatment-seeking problem gamblers will be allocated to either cognitive or exposure therapy in a two-group randomised, parallel design. Repeated measures will be conducted at baseline, mid and end of treatment (12 sessions intervention period), and at 3, 6 and 12 months (maintenance effects). The primary outcome measure is improvement in problem gambling severity symptoms using the Victorian Gambling Screen (VGS) harm to self-subscale. VGS measures gambling severity on an extensive continuum, thereby enhancing sensitivity to change within and between individuals over time.
\end{abstract}

Discussion: This article describes the research methods, treatments and outcome measures used to evaluate gambling behaviours, problems caused by gambling and mechanisms of change. This study will be the first randomised, parallel trial to compare cognitive and exposure therapies in this population.

Ethics and dissemination: The study was approved by the Southern Adelaide Health Service/Flinders University Human Research Ethics Committee. Study findings will be disseminated through peer-reviewed publications and conference presentations.

Trial registration: Australian New Zealand Clinical Trials Registry: ACTRN 12610000828022.

\section{INTRODUCTION}

Pathological gambling (PG) from a mental health perspective is defined by the American Psychiatric Association DSM-IV-TR as '...persistent and recurrent maladaptive gambling

\section{ARTICLE SUMMARY}

Article focus

- To isolate and compare cognitive and behavioural (exposure-based) techniques to determine their relative efficacy in treating problem gambling.

- The primary research question is: among treatment-seeking problem gamblers, is exposure therapy more effective in reducing gambling severity symptoms (harm to self-subscale of VGS) over the 15-month study period (intervention and maintenance effects) compared with cognitive therapy?

Key messages

- Problem gambling is a serious public health concern at an international level and occurs more frequently in younger populations.

- The best evidence for gambling treatments is for cognitive behavioural therapy but remains tentative.

- This study will be the first randomised, parallel trial to compare cognitive and exposure therapies in this population.

Strengths and limitations of this study

- A key strength of this study is that all treatmentseeking problem gamblers meeting eligibility criteria will receive active treatment. Also, owing to the broad study inclusion criteria, it is expected that a significant proportion of the sample will have comorbid conditions (eg, anxiety, depression and substance abuse which will enhance the external validity of findings using an intent-to-treat design.

- A limitation of the design is that there was no control group to account for non-specific treatment effects; however, a reasonable assumption is made that non-specific effects will be approximately similar between study groups due to the analogous therapy structures, therapist background and experience and therapeutic environment.

behaviour that disrupts personal, family and vocational pursuits'. ${ }^{1}$ The term 'problem gambling' defines less severe forms of gambling disorders and has been the basis for the development of several diagnostic and screening 
instruments such as the Canadian Problem Gambling Index (CGPI) ${ }^{2}$ and the Victorian Gambling Screen (VGS).$^{3}$ It is a serious public health concern at an international level where population prevalence rates average $2 \%$ or more and occurs more frequently in younger populations. ${ }^{4-9}$ Comorbid mental health disorders such as depression and anxiety are common in both treatmentseeking and general populations of problem gamblers. ${ }^{10}$

Treatment types available for gambling disorders have similarities to those of other addictions and include psychological, peer-support and, more recently, pharmacological interventions. ${ }^{11}{ }^{12}$ Until now, the best evidence for gambling treatments is psychological, where cognitive behavioural therapy (CBT) has been the most researched $^{13}$ and the evidence-base recommended as '...trusted to guide practice in most situations' based on the National Health and Medical Research Council grades for developers of guidelines. ${ }^{14}$

The theoretical underpinnings of CBT include cognitions (eg, erroneous thoughts) and psychobiological states (eg, physiological arousal) and are two dominant approaches to explaining gambling disorders. ${ }^{15}$ Most variants of CBT for treating gambling disorders have shown to be clinically beneficial compared with no treatment, and the most rigorous clinical trials have comprised a combined cognitive-behavioural approach. ${ }^{13} 16$ For core techniques, findings have indicated that cognitive therapy (CT) has an 'added advantage' when compared directly with no treatment, although evidence is tentative due to the heterogeneity between studies. ${ }^{13}$ Cognitive restructuring plays an important role in CT and has been shown to be beneficial in treating a range of mental health conditions. ${ }^{17}$

However, the comparative efficacy of CT with other core CBT interventions such as behavioural (exposurebased) therapies that target psychobiological-related gambling pathology is unknown. ${ }^{13}$ Exposure therapy (ET) is grounded in classical and operant conditioning paradigms and, where cue-exposure with extinction processes has been proposed as more beneficial than other types (eg, aversive therapy), in treating gambling addiction. ${ }^{18}$ ET has been shown to be as effective as CT in treating anxiety disorders where both techniques have similar hypothesised mechanisms of therapeutic change to those in gambling disorders. ${ }^{19}{ }^{20}$ In addictions, there is no conclusive evidence for exposure-based treatments due to there being few clinical trials with power. ${ }^{21}$

As behavioural therapies in general are more parsimonious in terms of delivery than $\mathrm{CT}^{22}$ it is important from a public-health point of view to understand the relative efficacy of these core treatments. Therefore, we designed a study titled 'Comparing outcomes of cognitive and exposure therapy for problem gambling', which is a randomised trial comparing the efficacies of CT and ET. This trial is motivated by the uncertainty about the clinical superiority of CT over ET. Based on this uncertainty, the concept of equipoise exists and participants will therefore not be disadvantaged from randomisation to either treatment group. This study will be the first to compare these treatments in a population of treatmentseeking gamblers.

\section{Study design}

Comparing the outcomes of cognitive and ET for gambling disorders is a two-group randomised, parallel design with treatment-seeking problem gamblers presenting to the Statewide Gambling Therapy Service (SGTS) in South Australia. The study will recruit 130 participants over a 12-month period starting April 2011. The outpatient SGTS programme offers one-on-one and group therapies for problem gamblers at three key metropolitan (Adelaide) centres, one of which will be selected as the trial site. The primary referral sources of clients presenting to SGTS are self, Gambling Helpline and related agencies and general practitioners. The service is staffed by a psychiatrist and therapists with professional registration in psychology, nursing or social work. All therapists have graduate qualifications and clinical experience in $\mathrm{CBT}^{19}$ Data collection will finish in July 2013.

The study was approved by the Southern Adelaide Health Service/Flinders University Human Research Ethics Committee and registered with the Australian New Zealand Clinical Trials Registry (ACTRN12610000828022) at the trials inception. The research officer will give participants an information statement regarding the study and ask for written informed consent before data collection begins.

\section{Participant recruitment and random assignment}

To assess study eligibility, an independent clinician will conduct semistructured interviews with treatmentseeking problem gamblers presenting to SGTS during the recruitment period. The interview will include assessment of individual demographics, recent gambling activities and administration of the well-validated South Oaks Gambling Screen (SOGS) ${ }^{23}$ SOGS is a 20 -item questionnaire based on DSM criteria for PG. A score of 5 or more is indicative of a probable pathological gambler. In gambling treatment samples, the scale has good reliability, exhibits high correlations with DSM-IV diagnostic criteria, and good-to-excellent classification accuracy. ${ }^{24}$

Study eligibility will be based on the following inclusion criteria: 18 years of age or older; treatment seeking for problem gambling with electronic gaming machines (EGMs); not involved in a concurrent gambling treatment programme; not received psychological treatment for problem gambling in the previous 12 months; willing to participate in the study; a willingness to read and respond to self-rated questionnaires written in English; willing to be randomised to one of two psychological treatments; gambled in the past month using EGMs; willing to provide follow-up data; willing to have treatment sessions audio recorded; scoring 5 or greater on SOGS and not suicidal or experiencing mental distress, such as mania, which would indicate that the problem 
gambler would not be able to participate fully in the treatment offered.

Individuals assessed as eligible for study participation will be randomly assigned to one of two treatment groups with a 1:1 allocation ratio. Randomisation will be blocked to increase the likelihood of equal group sizes, using a standard permutated block algorithm in which block sizes will be randomly chosen from 2, 4 and 6 to protect concealment. To ensure balance on potential confounders, block randomisation within strata will be used, stratifying at median age, gender and median SOGS scores for problem gambling severity. Based on previous SGTS data, age will be stratified as 18-42, and 43 years or more. ${ }^{25}$ Gambling severity will be stratified according to previous treatment-seeking problem gamblers' SOGS scores of either 5-11 or between 12 and $20{ }^{26} \mathrm{~A}$ statistician will independently generate random sequences for each stratum using Stata V.11.1 software $^{27}$ and deliver to the clinical trials call centre of a centrally located hospital pharmacy. Staff enrolling and referring participants, as well as those collecting and entering data and administering interventions, will not know in advance which treatment the next participant will receive.

\section{Sample size}

The primary research question is: among treatmentseeking problem gamblers, is ET more effective in reducing gambling severity symptoms (harm to self-subscale of the VGS) over the 12-month study period (intervention and maintenance effects) compared with CT?

Based on a type I error rate of $5 \%$, power of $90 \%$, twotailed test, and a VGS SD of 10.2 units, ${ }^{25}$ to detect a significant difference of $8 \%$ (ie, 4.8 points on the scale) in the mean VGS scores between the ET and CT groups, 50 participants will be required in each group. Given the treatment dropout rate experienced in the SGTS treatment programme (approximately 30\%), we therefore would need to recruit 65 participants in each group of the study, giving a total sample size of 130 participants.

\section{Therapists}

CT will be provided by two psychotherapists with qualifications in psychology and, on average, having approximately 5 years practice experience, including 2 years in treating individuals with gambling disorders. The therapists will receive initial on-site training in CT by Robert Ladouceur, a widely published international clinician and researcher in the field of CT for gambling disorders. $^{28-30}$

ET will be provided by two psychotherapists with postgraduate qualifications in CBT: a registered mental health nurse and a psychology graduate. On average, therapists have 6 years clinical experience in delivering CBT treatments to clients of SGTS, including a manualised ET programme. Therapists will receive on-site supervision from Malcolm Battersby who trained at the Institute of Psychiatry, London in behavioural treatments of anxiety disorders and severe neurotic conditions and is the Director of the Flinders Gambling Research Centre and SGTS. ${ }^{19}$

\section{Study treatments}

The trial will comprise two interventions:CT and ET. Participants in both groups will receive a standard of 12 60 min individual treatment sessions, ranging from 4 to 16 , depending on co-occurring conditions, conducted at weekly intervals. Both treatment manuals are intended as a session-by-session guide for therapists treating individuals with a gambling disorder where EGMs are the main form of gambling problem. The therapists will deliver treatment according to the content of each manual and sequencing of techniques in a face-to-face format. A summary of treatment sessions is provided in table 1 .

\section{Blinding}

Statistical analyses will be conducted according to prespecified guidelines. In this trial, therapists will know what treatment they are administering and participants will be provided with information that will rationalise and describe their assigned therapy protocol. Participants will be blinded to the study hypothesis in order to reduce the likelihood for self-report bias. Participant information sheets will refer to treatments as 'well known and commonly used psychological treatments'.

\section{Treatment integrity}

All treatment sessions will be audio recorded and $20 \%$ will be randomly selected from the early, mid and late study phases and evaluated using a checklist based on the CT Scale (table 2) ${ }^{31}$ ET sessions will be evaluated by $\mathrm{MB}$ and RP who are senior consultant psychiatrists with the Flinders Gambling Research Centre and have extensive experience in treatments for gambling disorders and other addictions ${ }^{19}{ }^{32}$ CT sessions will be evaluated by RL and MD who are senior clinical psychologists.

\section{Measures}

The administration of measures during the intervention period will be conducted prior to the start of each treatment session and at the 3-month and 6-month follow-up visits with a study therapist. The 12-month follow-up questionnaires will be mailed to participants. Baseline assessment will include demographic variables and data for the duration of the gambling problem. Validated outcome measures will cover domains of gambling behaviours, problems caused by gambling, and mechanisms of change. ${ }^{33}$ This means that, for ET participants, a greater reduction in the urge to gamble is expected to be associated with a clinically meaningful improvement in treatment outcomes than for CT participants. For CT participants, a more accurate set of beliefs relating to gambling is expected to be associated with a clinically meaningful improvement in treatment outcomes than for ET participants. The measures are summarised in 
Table 1 Intervention schedule

\begin{tabular}{|c|c|c|}
\hline $\begin{array}{l}\text { Weekly } \\
\text { sessions }\end{array}$ & Cognitive therapy (CT) & Exposure therapy (ET) \\
\hline Session 1 & $\begin{array}{l}\text { Pretreatment assessment to identify problem } \\
\text { gambling and any comorbid conditions. Rationale and } \\
\text { protocol of cognitive therapy explained }\end{array}$ & $\begin{array}{l}\text { Pretreatment assessment to identify problem } \\
\text { gambling and any comorbid conditions. Rationale and } \\
\text { protocol of exposure therapy explained }\end{array}$ \\
\hline Session 2 & $\begin{array}{l}\text { Development of participant's measurable problems } \\
\text { and goals. Analysis of a gambling session to identify } \\
\text { erroneous thoughts. Start a daily self-monitoring diary }\end{array}$ & $\begin{array}{l}\text { Development of participant's measurable problems } \\
\text { and goals. Establish cash restrictions to ensure } \\
\text { participant has no cash. First exposure task set using } \\
\text { images. Start a daily self-monitoring diary }\end{array}$ \\
\hline Session 3 & $\begin{array}{l}\text { Psychoeducation: clarification of the concept of } \\
\text { chance and establishing the distinction between } \\
\text { games of skill and games of chance }\end{array}$ & $\begin{array}{l}\text { Review participant's attempt at first exposure task. } \\
\text { Finalise cash restriction strategies if not already in } \\
\text { place. In-session imagery exposure task with } \\
\text { therapist guidance }\end{array}$ \\
\hline Session 4 & $\begin{array}{l}\text { Psychoeducation/cognitive awareness: introduce } \\
\text { ABCD (situation, thoughts, behaviour, consequences) } \\
\text { model and exercises to focus on the gambling } \\
\text { thoughts or 'inner dialogue' }\end{array}$ & $\begin{array}{l}\text { Review imagery exposure task. Finalise cash } \\
\text { restriction strategies if not already in place. Imagery } \\
\text { exposure task with therapist guidance }\end{array}$ \\
\hline Session 5 & $\begin{array}{l}\text { Identifying erroneous thoughts or 'gambling traps' that } \\
\text { lie behind emotions taking over reason using the } \\
\text { ABCD model. Participants are encouraged to } \\
\text { challenge these thoughts, perceptions and beliefs in } \\
\text { this session }\end{array}$ & $\begin{array}{l}\text { Review imagery exposure task. Introduction of next } \\
\text { exposure task involving image and sounds of } \\
\text { gambling-related cues }\end{array}$ \\
\hline Session 6 & $\begin{array}{l}\text { Identifying erroneous cognitions. Practical exercise to } \\
\text { help participant organise and act upon thoughts }\end{array}$ & $\begin{array}{l}\text { Introduction to the first of the in vivo exposure tasks. } \\
\text { This task to take place outside the participant's usual } \\
\text { gambling venue(s). The participant utilises principles } \\
\text { of exposure therapy from imaginal tasks to assist in } \\
\text { identifying what is happening to them at the time of } \\
\text { the in vivo task }\end{array}$ \\
\hline Session 7 & $\begin{array}{l}\text { Identifying erroneous cognitions. Practical exercise to } \\
\text { help participant organise and act upon thoughts } \\
\text { (continued) }\end{array}$ & $\begin{array}{l}\text { Fine-tuning of in vivo exposure task outside the } \\
\text { venue. Introduction to the in vivo exposure task to } \\
\text { take place inside the venue without cash }\end{array}$ \\
\hline Session 8 & $\begin{array}{l}\text { Develop skills for challenging and casting doubt on } \\
\text { the erroneous thoughts that lead to excessive } \\
\text { gambling }\end{array}$ & $\begin{array}{l}\text { Fine-tuning of in vivo exposure task inside the venue } \\
\text { without cash. Introduction to the next in vivo task } \\
\text { taking place inside a gambling venue with a small } \\
\text { amount of cash }\end{array}$ \\
\hline Session 9 & $\begin{array}{l}\text { Develop skills for challenging and casting doubt on } \\
\text { the erroneous thoughts that lead to excessive } \\
\text { gambling (continued) }\end{array}$ & $\begin{array}{l}\text { Fine-tuning of in vivo exposure task inside the venue } \\
\text { with a small amount of cash. Introduction to the next } \\
\text { in vivo task taking place inside a gambling venue } \\
\text { changing a small amount of cash for Poker machine } \\
\text { coins }\end{array}$ \\
\hline Session 10 & $\begin{array}{l}\text { Develop skills for challenging and casting doubt on } \\
\text { the erroneous thoughts that lead to excessive } \\
\text { gambling (continued) }\end{array}$ & $\begin{array}{l}\text { Review in vivo exposure tasks. Introduction to the } \\
\text { next in vivo task taking place inside a gambling } \\
\text { venue changing a small amount of cash for coins and } \\
\text { placing in the Poker machine }\end{array}$ \\
\hline $\begin{array}{l}\text { Sessions } 11 \\
\text { and } 12\end{array}$ & $\begin{array}{l}\text { Explore gambling relapse and develop relapse } \\
\text { prevention strategies }\end{array}$ & $\begin{array}{l}\text { Explore gambling relapse and develop relapse } \\
\text { prevention strategies }\end{array}$ \\
\hline
\end{tabular}

the following sections and the measurement occasions are presented in table 3 .

In order to detect changes in problem gambling severity during treatment and at follow-up, VGS will be utilised as a primary outcome measure. VGS is a self-reported questionnaire measuring the extent to which gambling behaviour has impeded an individual's life. The screen comprises three subscales (enjoyment of gambling, harm to partner and harm to self) with a total of 21 items. In this study, the harm to self-subscale will be used as the primary outcome measure. Items on the self-harm subscale relate to the person's experiences in the previous 4 weeks and have been validated for use in Australia in a clinical population of problem gamblers. ${ }^{34}$ Total scores for the 15 items range from $0=$ no harm to self to $60=$ high harm to self and a score of $21+$ identifies a person as a problem gambler. Concurrent validity indicates that the scale correlates very highly with SOGS but extends the score range.

Secondary outcome measures will include DSM-IV-TR diagnostic criteria relating to the extent of persistent and recurrent maladaptive gambling behaviour and be 
Table 2 Treatment integrity checklist items

\begin{tabular}{|c|c|c|c|}
\hline Item & $\begin{array}{l}\text { Response } \\
\text { options }\end{array}$ & Cognitive therapy & Exposure therapy \\
\hline 1 & $\begin{array}{l}\text { Yes/no/or N/A } \\
\text { (not applicable) }\end{array}$ & Eliciting automatic thoughts: gambling related & $\begin{array}{l}\text { Cash management: effective plan established } \\
\text { and agreed by the client }\end{array}$ \\
\hline 2 & Yes/no/or N/A & $\begin{array}{l}\text { Case conceptualisation: linking beliefs and } \\
\text { thoughts with behaviour, eliciting feedback from } \\
\text { client regarding validity and usefulness }\end{array}$ & $\begin{array}{l}\text { Case conceptualisation: linking autonomic } \\
\text { responses with behaviour, eliciting feedback } \\
\text { from client regarding validity and usefulness }\end{array}$ \\
\hline 3 & Yes/no/or N/A & $\begin{array}{l}\text { Sharing conceptualisation with client: used } \\
\text { meaningful examples }\end{array}$ & $\begin{array}{l}\text { Sharing conceptualisation with client: used } \\
\text { meaningful examples }\end{array}$ \\
\hline 4 & Yes/no/or N/A & Eliciting core beliefs/schemata: gambling related & $\begin{array}{l}\text { Eliciting autonomic symptoms, thoughts, and } \\
\text { behaviours: gambling related }\end{array}$ \\
\hline 5 & Yes/no/or N/A & $\begin{array}{l}\text { Addressing key issues: raised key issues and } \\
\text { related them to cognition and behaviour }\end{array}$ & $\begin{array}{l}\text { Setting and conduct of exposure tasks: } \\
\text { appropriately graded, focused, prolonged and } \\
\text { repeated; agreed by the client; relevant to } \\
\text { therapy goals }\end{array}$ \\
\hline 6 & Yes/no/or N/A & $\begin{array}{l}\text { Guided discovery: Socratic questioning, } \\
\text { reflective/confronting (eg, what would that } \\
\text { mean?)/interpretive responses to guide client's } \\
\text { understanding }\end{array}$ & $\begin{array}{l}\text { Addressing key issues: raised key issues and } \\
\text { related them to urge and behaviour }\end{array}$ \\
\hline 7 & Yes/no/or N/A & $\begin{array}{l}\text { Asking for alternative thoughts: alternative views/ } \\
\text { explanations appropriately followed through }\end{array}$ & $\begin{array}{l}\text { Habituation: evidence that the therapist assisted } \\
\text { the client to identify and habituate to } \\
\text { spontaneous urges }\end{array}$ \\
\hline 8 & Yes/no/or N/A & $\begin{array}{l}\text { Use of alternative cognitive techniques: } \\
\text { appropriately selected and applied, relevant to } \\
\text { therapy goals }\end{array}$ & $\begin{array}{l}\text { Use of alternative behavioural techniques: } \\
\text { appropriately selected and applied, relevant to } \\
\text { therapy goals }\end{array}$ \\
\hline 9 & $\begin{array}{l}0-10 \text {-Likert } \\
\text { scale }\end{array}$ & Overall rating of integrity & Overall rating of integrity \\
\hline 10 & $\begin{array}{l}\text { Unlimited free } \\
\text { form text }\end{array}$ & $\begin{array}{l}\text { Overall use of appropriate technique } \\
\text { (specifically, please comment on any area of the } \\
\text { session which may not have adhered to the } \\
\text { allocated therapeutic approach) }\end{array}$ & $\begin{array}{l}\text { Overall use of appropriate technique } \\
\text { (specifically, please comment on any area of the } \\
\text { session which may not have adhered to the } \\
\text { allocated therapeutic approach) }\end{array}$ \\
\hline
\end{tabular}

administered by study therapists. A total score is obtained by summing across the 10 responses of 'yes' or 'no'. A score of 5 or more indicates PG. ${ }^{1}$ All other secondary measures are self-reported. Gambling-related cognitions will be measured using a 23-item scale (GRCS) that records common thoughts associated with problem gambling, where each item is rated on a 1-7 Likert scale. ${ }^{35}$ The urge to gamble will be assessed using a 6-item instrument (GUS), where each item is rated on a 1-7 Likert scale. ${ }^{36} 37$ Participants' experiences of psychological distress will be recorded using a 10-item global measure (K10), with each item rated on a 1-5 Likert scale. ${ }^{38}$ To measure an individual's perspective of their functional ability/impairment relating to work and social activities, a 5-item scale (WSAS) will be used where each item is rated on a 0-8 Likert scale. ${ }^{39}$ Participant levels of alcohol use will be assessed based on a 10-item scale where questions 1-3 measure the quantity and frequency of alcohol use, questions 4-6 measure the possible dependence on alcohol and questions 7-10 measure alcohol-related problems. ${ }^{40}$

Following an explanation of treatment rationale and protocol in session one, participants will be asked to rate their confidence in treatment (from $0=$ extremely unconfident to $6=$ extremely confident) and belief in treatment logical (from $0=e x t r e m e l y$ illogical to $6=$ extremely logical) at the start of session two. At treatment completion, participants will be asked to rate their level of satisfaction with

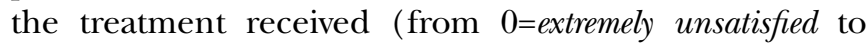
$6=$ extremely satisfied). To assess a participant's degree of confidence in their perceived ability to execute control of gambling behaviours during treatment and follow-up, a measure of self-efficacy will be utilised. Participants will describe up to three personally relevant high-risk situations and then rate the extent of their belief that they could refrain from gambling excessively in these situations on a scale of $0-10$. Self-reported measures of behaviours relating to problematic gambling will include: frequency of gambling in the previous month; number of hours spent on gambling activities in the previous month and amount spent on gambling activities in the previous month.

\section{Follow-up}

High rates of treatment and follow-up attrition occur for problem gamblers. ${ }^{41}$ To improve completion rates of self-rated questionnaires at follow-up for both treatment completers and treatment drop-outs, study participants will be offered honorarium gift vouchers to the value of $\$ 10$ at treatment completion; $\$ 20$ at 3-month follow-up; 


\section{Table 3 Measurements}

\begin{tabular}{|c|c|c|c|c|c|c|c|}
\hline \multirow[b]{2}{*}{ Measurements } & \multicolumn{4}{|c|}{ Intervention period } & \multicolumn{3}{|c|}{ Maintenance period } \\
\hline & Baseline & $\begin{array}{l}\text { Sessions } \\
2-12\end{array}$ & Mid-treatment & $\begin{array}{l}\text { End of } \\
\text { treatment }\end{array}$ & 3 months & 6 months & 12 months \\
\hline Demographics & \multicolumn{7}{|l|}{$x$} \\
\hline $\begin{array}{l}\text { Duration of gambling } \\
\text { problem }\end{array}$ & \multicolumn{7}{|l|}{$x$} \\
\hline AISS & \multicolumn{7}{|l|}{$X$} \\
\hline VGS & \multicolumn{2}{|l|}{$\mathrm{X}$} & $x$ & $x$ & $x$ & $x$ & $\mathrm{X}$ \\
\hline DSM-IV-TR & \multicolumn{2}{|l|}{$x$} & & \multirow{2}{*}{\multicolumn{2}{|c|}{$x$}} & \multicolumn{2}{|l|}{$x$} \\
\hline \multicolumn{6}{|l|}{ Mechanisms of change } & & \\
\hline GRCS & $x$ & $x$ & $x$ & $x$ & $x$ & $X$ & $x$ \\
\hline GUS & $x$ & $\mathrm{x}$ & $x$ & $x$ & $x$ & $\mathrm{X}$ & $x$ \\
\hline Self-efficacy & $\mathrm{x}$ & $\mathrm{x}$ & $\mathrm{x}$ & $x$ & $x$ & $x$ & $\mathrm{x}$ \\
\hline \multicolumn{8}{|c|}{ Problems caused by gambling } \\
\hline $\mathrm{K} 10$ & \multicolumn{3}{|l|}{$x$} & $x$ & $x$ & $x$ & $x$ \\
\hline WSAS & \multicolumn{3}{|l|}{$x$} & $x$ & $x$ & $x$ & $x$ \\
\hline AUDIT & \multicolumn{3}{|l|}{$x$} & $x$ & $x$ & $x$ & $x$ \\
\hline \multicolumn{8}{|l|}{ Gambling behaviours } \\
\hline Frequency ${ }^{\star}$ & \multicolumn{2}{|l|}{$x$} & $x$ & $x$ & $x$ & $x$ & $x$ \\
\hline Hourst & \multicolumn{2}{|l|}{$x$} & $x$ & $x$ & $x$ & $x$ & $x$ \\
\hline Amount $\ddagger$ & \multicolumn{2}{|l|}{$x$} & $x$ & $x$ & $x$ & $\mathrm{X}$ & $x$ \\
\hline \multicolumn{8}{|l|}{ Treatment views } \\
\hline $\begin{array}{l}\text { Confidence about } \\
\text { treatment }\end{array}$ & & $x \S$ & & & & & \\
\hline Treatment is logical & & $x \S$ & & & & & \\
\hline Satisfied with treatment & & & & $\mathrm{x}$ & & & \\
\hline
\end{tabular}

$\$ 25$ at 6-month follow-up and $\$ 30$ at 12-month follow-up. For follow-up assessments, a relatively large time window will be allowed to ensure maximum data collection and questionnaires will be administered by post to limit the burden to participants that may result from attending appointments at the study-site during follow-up. ${ }^{42}$

\section{Treatment drop-out}

Treatment drop-out will be determined using two general approaches. ${ }^{41}$ The first approach will be based on the therapists' judgement of participant progress up to the point of self-initiated termination. Second, attendance at a prespecified number of sessions will also be examined. A previous study involving SGTS participants engaged in ET determined an appropriate cut-off number of sessions for classification as drop-out as three or less, including the first screening attendance. ${ }^{25}$ Similarly, in a randomised trial evaluating CT, participants who attended an initial study screening and two treatment sessions or less were classified as drop-outs. ${ }^{29}$ The degree of concordance between these two classification methods for drop-outs will then be assessed and any discrepancies will be resolved by discussion between the clinical supervisors and therapists. The level of treatment adherence for each participant will help inform secondary data analyses discussed in the following section.

\section{Data analyses}

The primary analysis will be an intent-to-treat (ITT) to detect any statistically significant differences in VGS scores over time for the treatment and follow-up period between cognitive and ET. To account for participant attrition and lack of treatment adherence, the ITT analysis will be supplemented with an 'as treated' and 'per protocol' analysis. The 'as treated' approach will be useful for identifying associations between clusters of participant characteristics and treatment outcomes from an observational perspective. The 'per protocol' analysis will enable an evaluation of treatment efficacies in the subsample of participants who adhere to their assigned treatment protocol. ${ }^{43}$ Secondary measures will be analysed using the same approach as outlined above.

Generalised linear mixed models will be used for repeated measures of primary and secondary continuous and categorical outcomes. Preliminary models will assess for Therapist and Therapist X Treatment Group effects 
and will be included in the main analyses if statistically significant. Fixed effects in models will include the intervention group, time in continuous form (intervention period and maintenance effects) and interaction between group and time. Random effects will be at the study participant level and represent an upward or downward shift in the outcome measure from an overall regression line and the rate of change over time. Linear combinations of regression coefficients will be tested for treatment group effect at the completion of the intervention period and, for maintenance effects and estimates, will be presented along with CI. Predicted estimates of treatment outcomes at each time point will be calculated using fitted models of the data in order to examine patterns of individual change within each group. To interpret the effect sizes and precision for categorical outcomes, ORs and CI will be calculated.

To determine the mechanisms of therapeutic change based on the intended effects of each treatment, a mediation analysis will be conducted using mixed-effects modelling. The analysis will follow the traditional requirements for testing mediation: (1) testing for an association between treatment condition (ET and CT) and mediator (gambling urge or gambling-related cognitions);(2) testing for an association between treatment outcome variable (eg, gambling behaviours) and treatment condition; (3) testing for an association between the mediator and treatment outcome and(4) testing if the effect of treatment condition on treatment outcome is attenuated upon the addition of the mediator to the model. $^{44}$

In order to allow for an accurate critical appraisal of the validity and applicability of results, the reporting of this trial will comply with CONSORT (Consolidated Standards of Reporting Trials) guidelines for nonpharmacological treatment. ${ }^{45}$

\section{Qualitative component}

Despite the growing evidence base for cognitive and behavioural therapies for problem gambling, the processes and mechanisms of therapeutic change have not been explored from the participant's perspective and experience. Therefore, following the treatment intervention period, a subsample of participants will be invited to take part in semistructured interviews to explore therapeutic change for cognitive and exposure therapies. One-on-one interviews with participants will be conducted in person by the study research officer for approximately $1 \mathrm{~h}$ and audio recorded. To ensure the capture of a range of individual experiences, purposeful sampling will be used. Sampling will continue until theoretical saturation has been achieved, where no new or relevant data are seen to emerge. ${ }^{46}$ All recordings will be transcribed to a document, then verified for consistency by the research officer and uploaded to NVivo software $^{47}$ for data management. Interview transcripts will be analysed using direct content analysis to report the participant's experiences at the semantic level around specific and non-specific treatment effects. Initial coding will be conducted by the research officer and guided by existing theory and research. ${ }^{48} \mathrm{~A}$ second researcher will then cross-check the coding in order to reduce potential subjectivity in the analysis.

\section{DISCUSSION}

This study will provide high quality data for therapeutic benefits of ET compared with CT in people seeking treatment for problem gambling using EGMs. The outcome data collected will cover the domains of gambling behaviours, problems caused by gambling and mechanisms of change. Also, qualitative interviews will explore therapeutic change from the individual's experience and be examined in light of conclusions drawn from quantitative models to better understand each treatment's intended effects. The findings are expected to guide consumers, clinicians, policymakers and funders in the use of these treatments for gambling disorders.

The design of this trial is guided by ethical considerations, in line with the community service commitment of SGTS. Therefore, a key strength of this study is that all treatment-seeking problem gamblers meeting the eligibility criteria will receive active treatment. Also, owing to the broad study inclusion criteria, it is expected that a significant proportion of the sample will have comorbid conditions (eg, anxiety, depression and substance abuse $^{10}{ }^{49}$ ), which will enhance the external validity of findings using an ITT design. A limitation of the design is that there was no control group to account for nonspecific treatment effects; however, a reasonable assumption is made that non-specific effects will be approximately similar between study groups due to the analogous therapy structures, therapist background and experience and therapeutic environment.

A further limitation of the study design is that outcome data will be collected from self-report measures, and therefore participants may overestimate the treatment effects. As there is a high degree of uncertainty for differential treatment effects and blinding of participants to the study hypothesis, the likelihood of bias in self-ratings is expected to be minimised. Also, as this study will be conducted at a single site, the findings will have some limitations for inference to a wider population. On the other hand, the benefits of being a single-site study can include more effective lines of communication and a more consistent application of research protocol.

\section{CONCLUSION}

We have described the rationale and protocol for a randomised clinical trial to investigate the treatment efficacy of ET compared with CT for people with a gambling disorder in South Australia. The trial is funded, due to a limited gambling treatment evidence base, to inform a range of stakeholders on best practice. To our knowledge, this is the first randomised controlled trial to compare treatments with theoretical underpinnings 
from each of the two important approaches in explaining gambling disorders-cognitions (CT) and psychobiological states (ET) in treatment-seeking problem gamblers. The wide range of data collected in this trial will provide high-quality evidence for these treatments and contribute to the development of more optimal combinations of cognitive and behavioural therapies.

Acknowledgements Kirsten Dunn and Gaston Antezana are providing cognitive therapy. Jane Oakes and Amii Larsen are providing exposure therapy. Mitch Durbridge is providing on-site supervision for the cognitive therapy group and treatment integrity checks.

Contributors DPS, MWB, PWH, RGP and RL conceived the idea of the study and were responsible for the design of the study. The initial draft of the manuscript was prepared by DPS and MWB and then circulated repeatedly among all authors for critical revision. All authors read and approved the final manuscript.

Funding This work is funded by the Victorian Department of Justice, Office of Gaming \& Racing (tender 061/09), and management of the grant was transferred to the Victorian Responsible Gambling Foundation in July 2012. SGTS is funded by the Office of Problem Gambling, Department of Families and Social Inclusion, Government of South Australia. The authors alone are responsible for the design, implementation and reporting of results for this study.

Competing interests None.

Ethics approval Southern Adelaide Health Service/Flinders University Human Research Ethics Committee.

Provenance and peer review Not commissioned; internally peer reviewed.

\section{REFERENCES}

1. American Psychiatric Association. Diagnostic and statistical manual of mental disorders (4th Edition, Text Revision). 4th edn. Washington, DC, 2000

2. Ferris J, Wynne H. The Canadian problem gambling index. Ottawa, ON: Canadian Centre on Substance Abuse. 2001

3. Ben-Tovim D, Esterman A, Tolchard B, et al. The Victorian gambling screen: project report. Melbourne: Victorian Research Panel, 2001.

4. Becona E. Prevalence surveys of problem and pathological gambling in Europe: the cases of Germany, Holland and Spain. J Gambl Stud 1996;12:179-92.

5. Bondolfi G, Osiek C, Ferrero F. Prevalence estimates of pathological gambling in Switzerland. Acta Psychiatr Scand 2000;101:473-5.

6. Delfabbro P. Australasian gambling review. Independent Gambling Authority of South Australia, 2009.

7. Shaffer $\mathrm{HJ}$, Hall MN. Updating and refining prevalence estimates of disordered gambling behaviour in the United States and Canada. Can J Public Health 2001;92:168-72.

8. Wardle H, Sproston K, Orford J, et al. The British gambling prevalence survey. London: The Stationery Office, 2007.

9. Wong ILK, Ernest MTS. Prevalence estimates of problem and pathological gambling in Hong Kong. Am J Psychiatry 2003;160:1353

10. Lorains FK, Cowlishaw S, Thomas SA. Prevalence of comorbid disorders in problem and pathological gambling: systematic review and meta-analysis of population surveys. Addiction 2011;106(3): 490-8.

11. Daughters B, Lejuez CW, Lesieur HR, et al. Towards a better understanding of gambling treatment failure: implications of translational research. Clin Psychol Rev 2003;23:573-86.

12. Jackson A, Thomas S, Blaszczynski A. Best practice in problem gambling services. Melbourne: Gambling Research Panel, 2003.

13. Gooding P, Tarrier N. A systematic review and meta-analysis of cognitive-behavioural interventions to reduce problem gambling: hedging our bets? Behav Res Ther 2009;47:592-607.

14. Problem Gambling Research and Treatment Centre (PGRTC). Guideline for screening, assessment and treatment in problem gambling. Clayton: Monash University, 2011.

15. Clark L. Decision-making during gambling: an integration of cognitive and psychobiological approaches. Philos Trans $R$ Soc $B$ 2010;365:319.
16. Hodgins DC, Stea JN, Grant JE. Gambling disorders. Lancet 2011;378:1874-84.

17. Beck AT, Dozois DJA. Cognitive therapy: current status and future directions. Annu Rev Med 2011;62:397-409.

18. Brown RIF. Classical and operant paradigms in the management of gambling addictions. Behav Psychother 1987;15:111-22.

19. Battersby MW, Oakes J, Tolchard B, et al. Cognitive behavioural treatment for problem gamblers. In: Zangeneh M, Blaszczynski $A$, Turner NE, eds. In the pursuit of winning. New York: Springer, 2008:179-97.

20. Ougrin D. Efficacy of exposure versus cognitive therapy in anxiety disorders: systematic review and meta-analysis. BMC Psychiatry 2011;11:200.

21. Martin TD, LaRowe S, Malcolm R. Progress in cue exposure therapy for the treatment of addictive disorders: a review update. Open Addict J 2010;3:92-101.

22. Jacobson NS, Dobson KS, Truax PA, et al. A Component Analysis of Cognitive-Behavioral Treatment for Depression. J Consult Clin Psychol 1996;64:295-304.

23. Lesieur HR, Blume SB. The South Oaks Gambling Screen (SOGS): a new instrument for the identification of pathological gamblers. $A m$ J Psychiatry 1987;144:1184-8.

24. Stinchfield R. Reliability, validity, and classification accuracy of the South Oaks Gambling Screen (SOGS). Addict Behav 2002;27:1-19.

25. Smith D, Harvey $P$, Battersby $M$, et al. Treatment outcomes and predictors of drop out for problem gamblers in South Australia: a cohort study. Aust N Z J Psychiatry 2010;44:911-20.

26. Riley B, Smith D, Oakes J. Exposure therapy for pathological gambling in rural communities: a program model and early outcomes. Aust J Rural Health 2011;19:142-6.

27. StataCorp. Stata: Release 11. Statistical Software. College Station TX: StataCorp LP, 2009.

28. Ladoucer R, Sylvain C, Boutin C, et al. Cognitive treatment of pathological gambling. J Nerv Ment Dis 2001;189:774-80.

29. Ladouceur R, Sylvain C, Boutin C, et al. Group therapy for pathological gamblers: a cognitive approach. Behav Res Ther 2003;41:587.

30. Ladouceur R, Sylvain $\mathrm{C}$, Letarte $\mathrm{H}$, et al. Cognitive treatment of pathological gamblers. Behav Res Ther 1998;36:1111-19.

31. Vallis TM. The Cognitive Therapy Scale: psychometric properties. $J$ Consult Clin Psychol 1986;54:381-5.

32. Oakes J, Battersby M, Pols R, et al. Exposure therapy for problem gambling via videoconferencing: a case report. J Gambl Stud 2008;24:107-19.

33. Walker M, Toneatto T, Potenza MN, et al. A framework for reporting outcomes in problem gambling treatment research: the Banff, Alberta Consensus. Addiction 2006;101:504-11.

34. Tolchard B, Battersby M. The Victorian Gambling Screen: reliability and validity in a clinical population. J Gambl Stud 2010;26:623-38.

35. Raylu N, Oei T. The Gambling Related Cognitions Scale (GRCS): development, confirmatory factor validation and psychometric properties. Addiction 2004;99:757-69.

36. Raylu N, Oei T. The Gambling Urge Scale: development, confirmatory factor validation, and psychometric properties. Psychol Addict Behav 2004;18:100-5.

37. Smith DP, Pols RG, Battersby MW, et al. The Gambling Urge Scale: Reliability and validity in a clinical population. Addiction research \& theory 2013;21:113-22.

38. Andrews $\mathrm{G}$, Slade T. Interpreting scores on the Kessler psychological distress scale (K10). Aust N Z J Public Health 2001;25:494-7.

39. Mundt JC, Marks IM, Shear MK, et al. The work and social adjustment scale: a simple measure of impairment in functioning Br J Psychiatry 2002;180:461-4.

40. Reinert DF, Allen JP. The alcohol use disorders identification test (AUDIT): a review of recent research. Alcoholism 2002;26:272-9.

41. Melville KM, Casey LM, Kavanagh DJ. Psychological treatment dropout among pathological gamblers. Clin Psychol Rev 2007;27:944-58.

42. National Research Council. The prevention and treatment of missing data in clinical trials. Panel on handling missing data in clinical trials. Committee on National Statistics, Division of Behavioral and Social Sciences and Education. Washington, DC: The National Academies Press, 2010

43. Hernan MA, Hernandez-Diaz S. Beyond the intention-to-treat in comparative effectiveness research. Clin Trials 2012;9:48-55.

44. Baron RM, Kenny D. The moderator-mediator variable distinction in social psychological research: Conceptual, 
strategic, and statistical considerations. J Pers Soc Psychol 1986;51:1173-82.

45. Boutron IMDP, Moher DP, Altman DGD, et al. Extending the CONSORT statement to randomized trials of nonpharmacologic treatment: explanation and elaboration. Ann Intern Med 2008;148:295-309.

46. Crabtree B, DiCicco-Bloom B. The qualitative research interview. Med Educ 2006;40:314-21.
47. NVivo qualitative data analysis software. 10 edn. QSR International Pty Ltd, 2012.

48. Hsieh HF, Shannon SE. Three approaches to qualitative content analysis. Qual Health Res 2005;15:1277-88.

49. Ibanez A, Blanco C, Donahue E, et al. Psychiatric comorbidity in pathological gamblers seeking treatment. Am J Psychiatry 2001;158:1733-5 